\title{
Optimization of Demand Response Through Peak Shaving
}

\author{
G. Zakeri, D. Craigie ${ }^{\dagger}$ A. Philpott $;$ M. Todd ${ }^{\S}$
}

October 25, 2013

\begin{abstract}
We consider a consumer of a resource, such as electricity, who must pay a per unit charge to procure the resource, as well as a peak demand charge. We will assume that this consumer has some ability to self-generate and present an efficient linear programming formulation for the demand response of such a consumer. We will establish a monotonicity result that indicates fuel supply of $S$, utilized for self generation, may be spent in successive steps adding to $S$.
\end{abstract}

\section{Introduction}

We consider a model in which a consumer of a resource over several periods must pay a per unit charge for the resource as well as a peak usage charge. The consumer has the ability to reduce his consumption in any period at some given cost, subject to a constraint on the total amount of reduction possible. His problem is to decide in what periods to reduce his consumption to minimize the total cost of procuring the resource.

Such a model could arise in several settings. We have in mind an industrial or commercial consumer of electricity who uses a varying amount of electricity over some time horizon of $\mathcal{T}$ periods, for which he incurs an energy charge (per megawatt-hour consumed) and a peak usage charge for the maximum megawatt-hours consumed in the highest $k$ periods. The peak usage charge is otherwise known as a demand charge. The consumer has some onsite local generation that can be used to offset the purchases of electricity in any period. Such a charging regime is called anytime peak pricing or "Hopkinson rate" after the engineer who first proposed it in 1892 (see [3]).

A much simplified version of our problem was addressed in the late 1970s and early 1980s, before the prevalence of electricity markets, in the context of public utility pricing and rationing when demand exceeds the available supply (see for instance $[1,5,4]$ ). In this context, the authors attempt to deal with the details of rationing by assuming aggregate infinitesimal consumers that would provide a simple elastic demand curve with no constraints. This is a large point of difference from the setting that we face, where our consumer, possibly due to manufacturing constraints, is inflexible with respect to consumption of electricity. Furthermore, the above authors do not study properties (such as monotonicity) of their models.

Anytime peak pricing can be contrasted with coincident peak pricing (and its relation "time-ofuse" pricing) which imposes a demand charge in periods when the system experiences peak demand

${ }^{*}$ Corresponding author, Department of Engineering Science, University of Auckland, g.zakeri@auckland.ac.nz.

${ }^{\dagger}$ ASB Bank, London, UK.

${ }^{\ddagger}$ Department of Engineering Science, University of Auckland.

${ }^{\S}$ School of Operations Research and Information Engineering, Cornell University. 
(as modeled in [6] for example). The Hopkinson rate was originally intended to charge for electricity when it was primarily used for lighting, and so any user's peak demand typically coincided with the system peak. When these are different, it is not hard to see that coincident peak charging provides a clearer incentive to reduce the system costs incurred by increases in capacity. Notwithstanding this, anytime peak pricing does provide benefits from peak reduction (see e.g. [7]). It is also worth mentioning that for geographically isolated customers, coincident peak reduces to the Hopkinson rate.

Although the problem for a consumer facing an anytime peak charge is more straightforward than tackling the coincident peak problem, it is not trivial. The peak charge will typically be made on the total consumption over several periods, typically those $k$ periods with the largest consumption over some predetermined horizon. In this paper we show how these periods can be determined by a linear programming problem, to give an overall problem of minimizing cost that is also a linear program. This linear program is then shown to satisfy a monotonicity property that makes it amenable to solution by a greedy algorithm. This provides some insights into how to attack the problem with random data.

Although our problem might have applications in other settings we will couch it in the setting of electricity procurement. Nevertheless the analysis we develop is quite general.

The paper is laid out as follows. In the next section we formulate the optimization problem we will study, and show that it simplifies to a linear program. In Section 3 we show that this linear program has a specific structure that enables its solution by a greedy algorithm. The algorithm is outlined in Section 4; details can be found in [2].

\section{The anytime peak demand problem}

We start by defining the parameters and the variables of the problem. Throughout, we measure electricity in terms of the units of the fuel needed to produce it.

\section{Parameters}

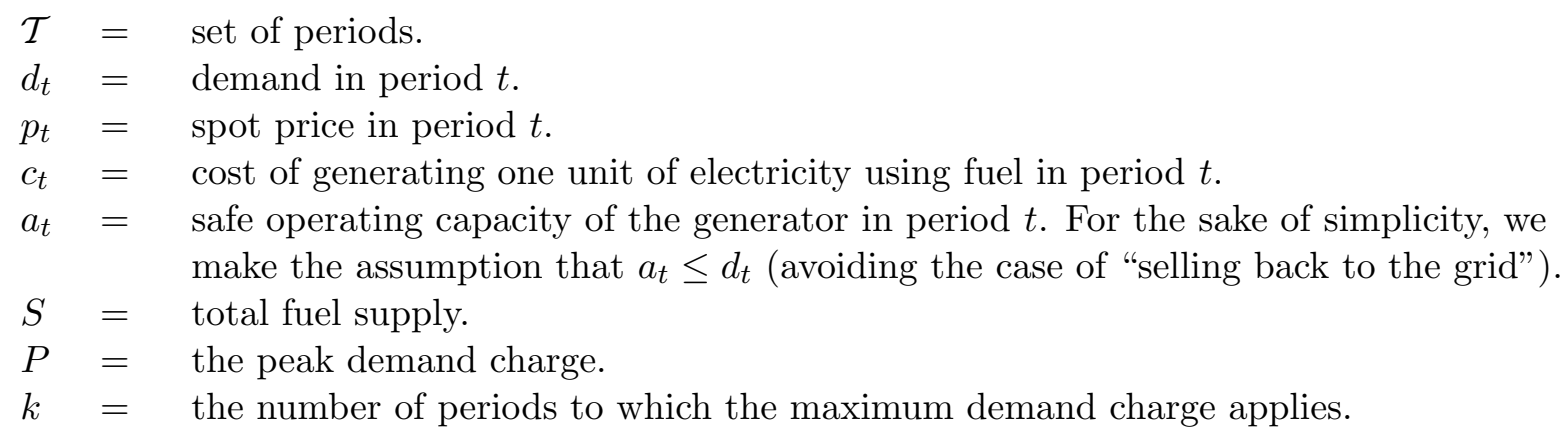

\section{Variables}

$s_{t}=$ amount of fuel to allocate to generation in period $t$.

$M=$ sum of the largest $k$ load realizations.

The consumer's problem over a time horizon $\mathcal{T}$ is to minimize the total cost of electricity consumed, plus the peak charges that are incurred on the top $k$ periods, while meeting every period's demand and employing a limited amount of self-generation. Without loss of generality we assume that $|\mathcal{T}| \geq k$. 
This problem can be formulated as:

$$
\begin{array}{llll}
{[\mathrm{AP}]: \min } & P M & +\sum_{t \in \mathcal{T}}\left(c_{t}-p_{t}\right) s_{t} & \\
\text { s.t. } & \sum_{t \in \mathcal{T}} s_{t} & \leq S & \\
& s_{t} & \leq a_{t} & t \in \mathcal{T} \\
& \sum_{t \in \mathcal{T}}\left(d_{t}-s_{t}\right) & \leq M & \text { for all } \tau \subseteq \mathcal{T},|\tau| \leq k .
\end{array}
$$

Note that there is no loss in generality in assuming that $P=1$ (by scaling the objective function of $[\mathrm{AP}])$, so we normalize the peak demand charge, i.e., set $P=1$, simply to make the presentation clearer.

Observe that in $[\mathrm{AP}]$ all subsets of $\mathcal{T}$ of size $k$ or less must be included which gives an exponentially growing set of constraints. The problem $[\mathrm{AP}]$ can be formulated more concisely using the following observation.

Given any feasible solution $s_{t}, t \in \mathcal{T}$, for [AP], the cost of maximum demand $M$ is the optimal value of

$$
\begin{array}{lllll}
{[\mathrm{MDP}]:} & \max & \sum_{t \in \mathcal{T}} \lambda_{t}\left(d_{t}-s_{t}\right) & & \\
& \text { s.t. } & \sum_{t \in \mathcal{T}} \lambda_{t} & \leq k, & \\
& \lambda_{t} & \geq 1 & t \in \mathcal{T} & {\left[y_{t}\right]} \\
& \lambda_{t} & & &
\end{array}
$$

Taking the dual of MDP gives

$$
\begin{array}{llllll}
{[\mathrm{MDD}]:} & \min & k h & +\sum_{t \in \mathcal{T}} y_{t} & & \\
& \text { s.t. } & h+y_{t} & \geq\left(d_{t}-s_{t}\right) & t \in \mathcal{T} & {\left[\lambda_{t}\right]} \\
& & y_{t} & \geq 0 & t \in \mathcal{T} &
\end{array}
$$

which has the same optimal value $M$. Here $M$ is the sum of the residual demands $d_{t}-s_{t}$ over the $k$ highest periods, which incurs penalty 1 . Henceforth we write $\forall t$ instead of $t \in \mathcal{T}$ for short.

In case $[\mathrm{MDP}]$ and $[\mathrm{MDD}]$ have multiple optimal solutions we need to focus on particular optimal solutions. Let us define $g(k)$ to be the $k$ th largest value of $d_{t}-s_{t}$ for $t \in \mathcal{T}$. We will then construct a set of periods that constitute the top $k$ periods (in terms of $d_{t}-s_{t}$ ), by resolving some ties. Define $\mathcal{N}=\left\{t \mid d_{t}-s_{t}>g(k)\right\}$. Now consider the set $\left\{t \mid d_{t}-s_{t}=g(k)\right\}$, order this set by $t$, and select the elements of $\mathcal{O}$ to be the first $k-|\mathcal{N}|$ periods in this (ordered) set. We will define $\mathcal{M}=\mathcal{N} \cup \mathcal{O}$. Note that $|\mathcal{M}|=k$, so we have determined a way of selecting "the top $k$ periods of residual demand" without ambiguity. We refer to $\mathcal{M}$ as our canonical maximum demand set. Note also that $\mathcal{M}$ depends on the vector $d-s$.

Lemma 1 For a given vector $d-s$, optimal solutions to [MDD] and [MDP] are given by

$$
\begin{gathered}
h^{*}=g(k) \\
y_{t}^{*}=\max \left(d_{t}-s_{t}-h^{*}, 0\right) \quad \forall t, \text { and } \\
\lambda_{t}^{*}= \begin{cases}P, & \text { if } t \in \mathcal{M}, \\
0, & \text { otherwise. }\end{cases}
\end{gathered}
$$

We refer to these solutions as the canonical solutions for residual demand $d-s$.

Proof. Observe that

$$
y_{t}^{*}= \begin{cases}d_{t}-s_{t}-h^{*}, & \text { if } t \in \mathcal{M}, \\ 0, & \text { otherwise. }\end{cases}
$$


Thus the optimality conditions for [MDP] and [MDD],

$$
\begin{array}{ll}
\sum_{t \in \mathcal{T}} \lambda_{t}=k & \\
0 \leq \lambda_{t} \leq 1 & \forall t \\
h+y_{t} \geq d_{t}-s_{t} & \forall t \\
y_{t} \geq 0 & \forall t \\
y_{t}\left(1-\lambda_{t}\right)=0 & \forall t \\
\lambda_{t}\left(h+y_{t}+s_{t}-d_{t}\right)=0 & \forall t,
\end{array}
$$

are satisfied by the solution in the statement of the lemma. Hence we have optimal solutions.

It is worth noting that [MDD] will almost always have an infinite number of solutions of which the canonical solution is only one. In fact for any $0 \leq \alpha \leq 1$,

$$
\begin{gathered}
h^{*}(\alpha)=\alpha g(k)+(1-\alpha) g(k+1), \\
y_{t}^{*}(\alpha)=\max \left(d_{t}-s_{t}-h^{*}(\alpha), 0\right) \quad \forall t, \text { and } \\
\lambda_{t}^{*}= \begin{cases}1, & \text { if } t \in \mathcal{M}, \\
0, & \text { otherwise. }\end{cases}
\end{gathered}
$$

will satisfy the optimality conditions of $[\mathrm{MDP}]$ and $[\mathrm{MDD}]$ and are therefore optimal.

Following $[\mathrm{MDP}]$ and $[\mathrm{MDD}]$, we can formulate $[\mathrm{AP}]$ as a linear program without having to consider an exponentially growing set of constraints. Define $f_{t}:=p_{t}-c_{t}$. The problem $[\mathrm{AP}]$ is equivalent to

$$
\begin{aligned}
& {[\mathrm{P}]: \min _{\text {s.t. }} k h+\sum_{t \in \mathcal{T}} y_{t}-\sum_{t \in \mathcal{T}} f_{t} s_{t}} \\
& \text { s.t. } \quad \sum_{t \in \mathcal{T}} s_{t} \leq S \quad[-\pi] \\
& h+y_{t}+\quad \begin{array}{rrrr}
s_{t} & \leq a_{t} \quad \forall t & {\left[-\eta_{t}\right.} \\
s_{t} & \geq d_{t} \forall t & {\left[\lambda_{t}\right]}
\end{array} \\
& y_{t}, \quad s_{t} \geq 0 \quad \forall t \text {. }
\end{aligned}
$$

Problem $[\mathrm{P}]$ can also be equivalently viewed as a bi-level LP

$$
\begin{array}{lll}
{[\mathrm{P}]:} & \min & -\sum_{t \in \mathcal{T}} f_{t} s_{t}+Q(s) \\
& \text { s.t. } & \sum_{t \in \mathcal{T}} s_{t} \leq S \\
& & 0 \leq s_{t} \leq a_{t}
\end{array} \quad \forall t
$$

where

$$
\begin{aligned}
& \text { [Stage2]: } Q(s):=\min k h+\sum_{t \in \mathcal{T}} y_{t} \\
& \text { s.t. } h+y_{t}+s_{t} \geq d_{t} \quad \forall t \quad\left[\lambda_{t}\right] \\
& y_{t} \geq 0 \quad \forall t .
\end{aligned}
$$

It is easy to see from Lemma 1 that for a fixed vector $s$, an optimal solution to [Stage2] is given by

$$
\begin{gathered}
h^{*}=g(k) \\
y_{t}^{*}=\max \left(d_{t}-s_{t}-h^{*}, 0\right) \quad \forall t .
\end{gathered}
$$




\section{Monotonicity properties}

In this section we explore the monotonicity properties of the solution of $[\mathrm{P}]$ as a function of the total fuel resource limit $S$. The optimality conditions for $[\mathrm{P}]$ are given by

$$
\begin{aligned}
& \text { [Primal Feasibility] } \quad h+y_{t}+s_{t} \geq d_{t} \quad \forall t \quad(\mathrm{PF} 1) \\
& s_{t} \leq a_{t} \quad \forall t \quad(\mathrm{PF} 2) \\
& \sum_{t \in \mathcal{T}} s_{t} \leq S \quad \text { (PF3) } \\
& y_{t}, s_{t} \geq 0 \quad \forall t \quad(\mathrm{PF} 4) \\
& \text { [Dual Feasibility] } \sum_{t \in \mathcal{T}} \lambda_{t}=k \quad \text { (DF1) } \\
& \lambda_{t} \leq 1 \quad \forall t \quad(\mathrm{DF} 2) \\
& \pi+\eta_{t}-\lambda_{t} \geq f_{t} \quad \forall t \quad \text { (DF3) } \\
& \lambda_{t}, \eta_{t}, \pi \geq 0 \quad \forall t \quad(\mathrm{DF} 4) \\
& \text { [Complementarity] } \quad \lambda_{t}\left(h+y_{t}+s_{t}-d_{t}\right)=0 \quad \forall t \\
& \eta_{t}\left(a_{t}-s_{t}\right)=0 \quad \forall t \\
& \pi\left(S-\sum_{t \in \mathcal{T}} s_{t}\right)=0 \\
& y_{t}\left(1-\lambda_{t}\right)=0 \quad \forall t \\
& s_{t}\left(\pi+\eta_{t}-\lambda_{t}-f_{t}\right)=0 \quad \forall t .
\end{aligned}
$$

Consider an amount of fuel $S$ to be allocated optimally to the periods in $\mathcal{T}$. Let $S=S^{1}+S^{2}$ with $S^{1}, S^{2}>0$ and let us introduce the following two problems.

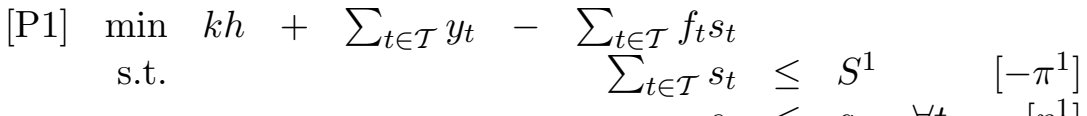

$$
\begin{aligned}
& h+y_{t}+\quad \begin{array}{llll}
s_{t} & \leq a_{t} & \forall t & {\left[\eta_{t}^{1}\right]} \\
s_{t} & \geq d_{t} \quad \forall t \quad\left[\lambda_{t}^{1}\right]
\end{array} \\
& y_{t}, \quad s_{t} \geq 0 \quad \forall t \text {. } \\
& \begin{array}{r}
\text { [P2] } \min k h+\sum_{t \in \mathcal{T}} y_{t}-\sum_{t \in \mathcal{T}} f_{t} s_{t} \\
\text { s.t. }
\end{array} \\
& h+\quad y_{t}+\quad \begin{array}{llll}
s_{t} \leq a_{t}-s_{t}^{1} & \forall t & {\left[\eta_{t}^{2}\right]} \\
s_{t} \geq d_{t}-s_{t}^{1} & \forall t & {\left[\lambda_{t}^{2}\right]}
\end{array}
\end{aligned}
$$

Note that in the definition of [P2], we use $s_{t}^{1}$ which is optimal for [P1]. In the next set of results, we prove that the optimal expenditure of the total fuel supply $S=S^{1}+S^{2}$ is equivalent to utilizing $S^{1}$ optimally first then continuing from there with the additional $S^{2}$ amount. This shows that the optimal $s_{t}$ 's are monotonic in $S$.

Lemma 2 Suppose that $s_{t}^{1}, y_{t}^{1}, h^{1}$ solve [P1] and that $\sum_{t \in \mathcal{T}} s_{t}^{1}<S_{1}$. Then $\hat{h}=h^{1}, \hat{y}_{t}=y_{t}^{1}$, and $\hat{s}_{t}=s_{t}^{1}$ is an optimal solution for $[P]$, and $h^{2}=h^{1}, y_{t}^{2}=y_{t}^{1}$, and $s_{t}^{2}=0$ is an optimal solution for [P2].

\section{Proof.}

Suppose that $\sum_{t \in \mathcal{T}} s_{t}^{1}<S^{1}$ where $\lambda_{t}^{1}, \eta_{t}^{1}$, and $\pi^{1}$ comprise an optimal dual solution for [P1]. The problem set up of [P1] and [P2], together with $S_{1}+S_{2}=S$ imply primal and dual feasibility of the left hand sides for problem $[\mathrm{P}]$. Notice also that complementarity conditions (CS1), (CS2), (CS4) and 
(CS5) clearly hold. To prove (CS3), we note that $\sum_{t \in \mathcal{T}} s_{t}^{1}<S_{1}$, hence to obtain (CS3) for problem [P1], it must be that $\pi^{1}=0$. This clearly yields (CS3) for [P].

Furthermore, $h^{2}=h^{1}, y_{t}^{2}=y_{t}^{1}$, and $s_{t}^{2}=0$ together with $\lambda_{t}^{2}=\lambda_{t}^{1}, \eta_{t}^{2}=\eta_{t}^{1}$, and $\pi^{2}=\pi^{1}=0$ constitute optimal solutions (and duals) for [P2] as these satisfy the optimality conditions of [P2].

Lemma 3 Consider the canonical solutions of [P1] and [P2] with residual demands $d-s^{1}$ and $d-$ $s^{1}-s^{2}$ respectively. Suppose that there exists $t$ such that $s_{t}^{2}>0$. Then there exists a period $\bar{t}$ such that $s_{\bar{t}}^{2}>0$ and $\lambda_{\bar{t}}^{1} \geq \lambda_{\bar{t}}^{2}$.

Proof. Let $\mathcal{M}^{1}$ and $\mathcal{M}^{2}$ be canonical maximum demand sets, as introduced in the previous section, for residual demands $d-s^{1}$ and $d-s^{1}-s^{2}$ respectively. If $t \in \mathcal{M}^{1}$ then $\lambda_{t}^{1}=1$, and so the lemma is proved with $\bar{t}=t$. If $t \notin \mathcal{M}^{1}$ and $t \notin \mathcal{M}^{2}$ then $\lambda_{t}^{1}=0$ and $\lambda_{t}^{2}=0$, so again the lemma is proved with $\bar{t}=t$.

We now focus on the case where $t \in \mathcal{M}^{2} \backslash \mathcal{M}^{1}$. Consider all $r \in \mathcal{M}^{1}$.

1. If $s_{r}^{2}=0 \quad \forall r \in \mathcal{M}^{1}$, then for all such $r,\left(d-s^{1}-s^{2}\right)_{r}=\left(d-s^{1}\right)_{r}$ so these remain the $k$ largest values, i.e., $\mathcal{M}^{1}=\mathcal{M}^{2}$ contradicting the existence of $t$.

2. Otherwise, there exists $r \in \mathcal{M}^{1}$ with $s_{r}^{2}>0$. In this case the lemma is proved by setting $\bar{t}=r$.

We can now show that the marginal value of fuel cannot increase as we move from the canonical solution of $[\mathrm{P} 1]$ to that of $[\mathrm{P} 2]$.

Lemma 4 The canonical solutions of [P1] and [P2] give $\pi^{2} \leq \pi^{1}$.

Proof. If

$$
\sum_{t \in \mathcal{T}} s_{t}^{2}<S^{2}
$$

then $\pi^{2}=0$ by (CS3), which gives the result. Otherwise there is some $t \in \mathcal{T}$ with $s_{t}^{2}>0$, which by Lemma 3 may be chosen without loss of generality so that $\lambda_{t}^{1} \geq \lambda_{t}^{2}$. Since $s_{t}^{2}>0$, we must have had $s_{t}^{1}<a_{t}$, and so $\eta_{t}^{1}=0$. Thus (DF3) for [P1] gives

$$
\pi^{1} \geq f_{t}+\lambda_{t}^{1} .
$$

Furthermore, since $s_{t}^{2}>0$, (CS5) for [P2] provides

$$
\pi^{2}=f_{t}+\lambda_{t}^{2}-\eta_{t}^{2} \leq f_{t}+\lambda_{t}^{2} \leq f_{t}+\lambda_{t}^{1}
$$

Equations (4) and (5) yield $\pi^{2} \leq \pi^{1}$.

Lemma 5 Suppose that $s_{t}^{1}, y_{t}^{1}, h^{1}$ and $s_{t}^{2}, y_{t}^{2}, h^{2}$ are canonical solutions to [P1] and [P2] respectively. If $\sum_{t \in \mathcal{T}} s_{t}^{1}=S_{1}$ then $\hat{h}=h^{2}, \hat{y}_{t}=y_{t}^{2}$, and $\hat{s}_{t}=s_{t}^{1}+s_{t}^{2}$ is an optimal solution for [P].

Proof. Recall from Lemma 1 that

$$
\begin{gathered}
h^{1}=g^{1}(k) \\
y_{t}^{1}=\max \left(d_{t}-s_{t}^{1}-h^{1}, 0\right) \quad \forall t, \text { and }
\end{gathered}
$$




$$
\lambda_{t}^{1}= \begin{cases}1, & \text { if } t \in \mathcal{M}^{1} \\ 0, & \text { otherwise }\end{cases}
$$

where $g^{1}(k)$ denotes the size of the $k$ th largest element of $d^{1}-s^{1}$ and $\mathcal{M}_{1}$ denotes the canonical maximum demand set for the vector $d^{1}-s^{1}$. Similarly,

$$
\begin{gathered}
h^{2}=g^{2}(k), \\
y_{t}^{2}=\max \left(d_{t}-s_{t}^{1}-s_{t}^{2}-h^{2}, 0\right) \quad \forall t, \text { and } \\
\lambda_{t}^{2}= \begin{cases}1, & \text { if } t \in \mathcal{M}_{2}, \\
0, & \text { otherwise, }\end{cases}
\end{gathered}
$$

where $g^{2}(k)$ is the size of the $k$ th largest value of vector $d-s^{1}-s^{2}$ and $\mathcal{M}_{2}$ denotes the canonical maximum demand set for the vector $d-s^{1}-s^{2}$.

For $[\mathrm{P} 2]$, for any $t$ such that $s_{t}^{1}=a_{t}$, we have a degenerate solution (since $\left.s_{t}^{2}=0=a_{t}-s_{t}^{1}\right)$. In this case we specify $\eta_{t}^{2}$ as follows

$$
\eta_{t}^{2}=\max \left(\lambda_{t}^{2}+f_{t}-\pi^{2}, 0\right) \quad \forall t
$$

Clearly the optimality conditions of [P2] are satisfied with the above.

Now recall that $\sum_{t \in \mathcal{T}} s_{t}^{1}=S_{1}$ and we set

$$
\begin{array}{ll}
\hat{h}=h^{2} & \\
\hat{y}_{t}=y_{t}^{2} & \forall t \\
\hat{s}_{t}=s_{t}^{1}+s_{t}^{2} & \forall t \\
\hat{\lambda}_{t}=\lambda_{t}^{2} & \forall t \\
\hat{\eta}_{t}=\eta_{t}^{2} & \forall t \\
\hat{\pi}=\pi^{2} . &
\end{array}
$$

Similar to the argument for Lemma 2, it is clear that primal and dual feasibility conditions for $[\mathrm{P}]$ naturally follow from the optimality of problem [P2] at the stated solution. Also conditions (CS1), (CS2) and (CS4) follow immediately. To see (CS3), note here that optimality of [P2] at the solution above implies $\pi^{2}\left(S_{2}-\sum_{t \in \mathcal{T}} s_{t}^{2}\right)=0$. Since here we have $\sum_{t \in \mathcal{T}} s_{t}^{1}=S_{1}$, it follows that $\pi^{2}\left(S-\sum_{t \in \mathcal{T}}\left(s_{t}^{1}+s_{t}^{2}\right)=\pi^{2}\left(S_{2}-\sum_{t \in \mathcal{T}} s_{t}^{2}\right)=0\right.$.

It remains to ensure that

$$
\hat{s}_{t}\left(\pi^{2}+\eta_{t}^{2}-\lambda_{t}^{2}-f_{t}\right)=0
$$

is satisfied for all $t$. Here again if $s_{t}^{2}>0$, we must have had, from the optimality of [P2], that $\pi^{2}+\eta_{t}^{2}-\lambda_{t}^{2}-f_{t}=0$ and this clearly yields (CS5) for problem [P] as well. What is left to do is to examine all $t$ for which $s_{t}^{2}=0$. If $s_{t}^{1}=0$ as well then (CS5) is immediately established since $\hat{s}_{t}=s_{t}^{1}+s_{t}^{2}=0$.

We will establish (CS5) by considering the two remaining cases where $0<s_{t}^{1}<a_{t}$, and $s_{t}^{1}=a_{t}$, separately.

Case I $\left(0<s_{t}^{1}<a_{t}\right)$

Consider $t$ such that $0<s_{t}^{1}<a_{t}$, and $s_{t}^{2}=0$. From (CS2) for problem [P1] then we have that $\eta_{t}^{1}=0$. Furthermore since $s_{t}^{2}=0<a_{t}-s_{t}^{1}$ we also have $\eta_{t}^{2}=0$. 
Now $\eta_{t}^{1}=0$, together with $(\mathrm{CS} 5)$ for $[\mathrm{P} 1]$ yields that

$$
\pi^{1}=f_{t}+\lambda_{t}^{1} .
$$

Therefore by the choice of our canonical optimal solutions outlined above, we will have:

$$
\pi^{1}= \begin{cases}f_{t}+1, & \text { if } t \in \mathcal{M}^{1} \\ f_{t}, & \text { otherwise }\end{cases}
$$

1. Case I.1: Suppose $t \in \mathcal{M}^{1}$. Then $\pi^{1}=f_{t}+1$.

Since $s_{t}^{2}=0$, we must have $t \in \mathcal{M}^{2}$, because $t$ was a highest peak after $s_{t}^{1}$ and must remain so if no further reduction in load is made. Therefore $\lambda_{t}^{1}=\lambda_{t}^{2}=1$. Also, $\eta_{t}^{2}=0$ and (DF3) for [P2] provides

$$
\pi^{2} \geq f_{t}+1=\pi^{1}
$$

By Lemma 4 we get $\pi^{2}=\pi^{1}$ so

$$
\pi^{2}+\eta_{t}^{2}-\lambda_{t}^{2}-f_{t}=\pi^{1}+\eta_{t}^{1}-\lambda_{t}^{1}-f_{t}=0
$$

and thus (CS5) is established.

2. Case I.2: Suppose now that $t \notin \mathcal{M}^{1}$, so that $\lambda_{t}^{1}=0$ and from (6) $\pi^{1}=f_{t}$. If $t \notin \mathcal{M}^{2}$ then $\lambda_{t}^{2}=0$. Then (DF3) for [P2] will give

$$
\pi^{2} \geq f_{t}=\pi^{1}
$$

Lemma 4 yields $\pi^{2}=\pi^{1}$ so

$$
\pi^{2}+\eta_{t}^{2}-\lambda_{t}^{2}-f_{t}=\pi^{1}+\eta_{t}^{1}-\lambda_{t}^{1}-f_{t}=0
$$

and thus (CS5) is established. If on the other hand $t \in \mathcal{M}^{2}$ then $\lambda_{t}^{2}=1$. However (DF3) then yields

$$
\pi^{2} \geq f_{t}+1>f_{t}=\pi^{1}
$$

contradicting Lemma 4 .

Case II $\left(s_{t}^{1}=a_{t}\right)$

Lastly we must consider $t$ such that $s_{t}^{1}=a_{t}$, and $s_{t}^{2}=0$. Conditions (DF3) and (DF4) give

$$
\eta_{t}^{2}=\max \left(\lambda_{t}^{2}+f_{t}-\pi^{2}, 0\right)
$$

If $\eta_{t}^{2}=\lambda_{t}^{2}+f_{t}-\pi^{2}$ then clearly (CS5) for [P] is established.

If on the other hand $\eta_{t}^{2}=0$ then (DF3) for [P2] provides $\pi^{2} \geq f_{t}+\lambda_{t}^{2}$. However (CS5) for [P1] gives $\pi^{1}=f_{t}+\lambda_{t}^{1}-\eta_{t}^{1} \leq f_{t}+\lambda_{t}^{1}$.

Now if $\lambda_{t}^{1}>\lambda_{t}^{2}$ then we must have $t \in \mathcal{M}^{1}$ but $t \notin \mathcal{M}^{2}$. However this cannot happen as $s_{t}^{2}=0$ (see the argument in Case I.1).

Therefore it must be that $\lambda_{t}^{1} \leq \lambda_{t}^{2}$, which gives $\pi^{1} \leq f_{t}+\lambda_{t}^{1} \leq f_{t}+\lambda_{t}^{2} \leq \pi^{2}$ and so by Lemma 4 $\pi^{1}=\pi^{2}$ and $\lambda_{t}^{1}=\lambda_{t}^{2}$. It follows that $f_{t}+\lambda_{t}^{2}-\pi^{2}=0$ so $\eta_{t}^{2}=f_{t}+\lambda_{t}^{2}-\pi^{2}$ giving (CS5) for [P].

Theorem 6 The canonical solutions to [P1] and [P2] provide an optimal (canonical) solution to [P].

Proof. The proof follows immediately from Lemmas 2 and 5. 


\section{The algorithm}

The greedy peak shaving algorithm outlined below is detailed in chapter 5 of [2]. Assume without loss of generality that the periods are ordered by decreasing spot price and $s_{t}<\min \left\{a_{t}, d_{t}\right\}$ for all $t$. The optimal marginal allocation of fuel will be to a subset $\psi$ of periods: this is either the period(s) with highest price if none of these are in $\chi=\left\{t \mid d_{t}-s_{t}=g(k)\right\}$, or to a subset of $\chi$ consisting of its first $l$ periods for some $l$. We choose $\psi$ with the largest savings per unit of fuel allocated. Observe that this involves computing savings for at most $|\chi|+1$ sets. The algorithm then allocates fuel in equal increments to periods in $\psi$ until $s_{t}=\min \left\{a_{t}, d_{t}\right\}$, for some $t \in \psi$, or $\chi$ changes. Formally, the algorithm performs the following steps.

\section{Peak Shaving Algorithm}

While fuel quantity $S>0$ and $\psi$ is non-empty

1. Compute $\psi$ with largest savings;

2. Allocate fuel to all periods $t$ in $\psi$ until $s_{t}=\min \left\{a_{t}, d_{t}\right\}$, for some $t \in \psi$, or $\chi$ changes;

3. Decrease $S$ by fuel allocated;

4. If $s_{t}=\min \left\{a_{t}, d_{t}\right\}$ remove $t$ from the problem and update $\chi$.

\section{References}

[1] G. Brown and M. B. Johnson. Public utility pricing and output under risk. American Economic Review, 59:119-128, 1969.

[2] D. N. Craigie. Optimization of demand side electricity generation. Master's thesis, Department of Engineering Science, University of Auckland, 7 2007. Available online from www.epoc.org.nz/theses.html.

[3] J.L. Neufeld. Price discrimination and the adoption of the electricity demand charge. The Journal of Economic History, 47(3):693-709, 1987.

[4] S. S. Oren and S. A. Smith. Design and management of curtailable electricity service to reduce annual peaks. Operations Research, 40(2):213-228, 1992.

[5] J. C. Panzar and D. S. Sibley. Public utility pricing and output under risk: The case of selfrationing. American Economic Review, 68:888-895, 1978.

[6] A.B. Philpott and G. Pritchard. An electricity procurement model with energy and peak charges. In W.T. Ziemba, S.W. Wallace, and H. Gassman, editors, Stochastic Programming: Applications in Finance, Energy, Planning and Logistics. World Scientific, Singapore, 2013.

[7] M.R. Veall. Industrial electricity demand and the Hopkinson rate: An application of the extreme value distribution. The Bell Journal of Economics, 14(2):427-440, 1983. 